\title{
Cambios macroscópicos de la amalgama dental sometida in vitro a altas temperaturas con fines forenses: el caso de los nódulos de plata
}

\author{
Macroscopic Changes of Dental Amalgam subjected in vitro at High \\ Temperatures with Forensic Purposes: The Case of Silver Bullets
}

Gustavo Sinisterra-Sinisterra Odontólogo, Pontificia Universidad Javeriana, Bogotá, Colombia. Profesor de la Escuela de Odontología, Universidad del Valle, Cali, Colombia.

Liliana Marín-Jiménez Odontóloga, Institución Universitaria Colegios de Colombia, Bogotá,

Colombia. Magistra en Criminalística,

Universidad Libre, Cali, Colombia. Odontóloga forense del Cuerpo Técnico Investigativo de la Fiscalía General de la Nación, Bogotá, Colombia.

Angélica García Química, PhD en Ciencias-Química, Universidad del Valle, Cali, Colombia.

Profesora del Departamento de Ciencias Básicas de la Salud, Facultad de Ciencias de la Salud, Pontificia Universidad Javeriana, Cali, Colombia.

Sandra Moreno-Correa Odontóloga, magistra en Ciencias Biomédicas, Universidad del Valle,

Cali, Colombia. Profesora del Departamento de Ciencias Básicas de la Salud, Facultad de Ciencias de la Salud, Pontificia Universidad Javeriana, Cali, Colombia.

Freddy Moreno-Gómez Odontólogo, magíster en Ciencias Biomédicas, Universidad del Valle, Cali, Colombia. Profesor del Departamento de Ciencias Básicas de la Salud, Facultad de Ciencias de la Salud, Pontificia Universidad Javeriana, Cali, Colombia.

CÓMO CITAR ESTE ARTÍCULO Sinisterra-Sinisterra G, Marín-Jiménez L, García A. Moreno-Correa S, Moreno-Cómez F. Cambios macroscópicos de la amalgama dental sometida in vitro a altas temperaturas con fines forenses: el caso de las balas de plata. Univ Odontol. 2016 Ene-Jun; 35(74): 39-48. http://dx.doi. org/10.11144Javeriana.uo35-74.cmad

Recibido para publicación: 25/03/2015 Aceptado para publicación: 17/02/2016

Disponible en: http://www.javeriana.edu.co/ universitasodontologica

\section{RESUMEN}

Antecedentes: Los materiales de uso odontológico, como la amalgama dental, sufren cambios macroestructurales cuando se someten a altas temperaturas. Es posible emplear dichos cambios para determinar el tipo de material y estimar la temperatura máxima alcanzada durante los procesos de identificación odontológica y documentación de la necropsia médico-legal en casos de individuos quemados, carbonizados o incinerados. Objetivo: Describir la formación de nódulos de plata en superficies de amalgama dental expuestas a altas temperaturas para establecer parámetros y diseñar un marcador fehaciente de uso en odontología forense. Métodos: Se realizó un estudio cuasi experimental in vitro que describió la formación de nódulos de plata en la superficie de 45 discos de amalgama dental

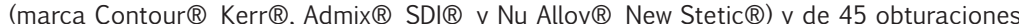
oclusales en premolares sometidos a altas temperaturas $\left(200{ }^{\circ} \mathrm{C}, 400{ }^{\circ} \mathrm{C}, 600^{\circ} \mathrm{C}\right.$ y $800{ }^{\circ} \mathrm{C}$ ). Resultados: Los nódulos de plata en los discos y en las obturaciones se formaron en la superficie de la amalgama desde los $200^{\circ} \mathrm{C}$ hasta los $400{ }^{\circ} \mathrm{C}$ y empezaron a desaparecer a los $600{ }^{\circ} \mathrm{C}$ para formar esferas y vetas hacia los $800{ }^{\circ} \mathrm{C}$. Conclusiones: La aparición y desaparición de los nódulos de plata es una característica repetitiva de las amalgamas dentales de última generación expuestas a diferentes temperaturas extremas. Por tal razón, su descripción puede emplearse como un marcador para identificar el biomaterial y determinar la temperatura máxima alcanzada por restos humanos.

\section{PALABRAS CLAVE}

altas temperaturas; amalgama dental; balas de plata; ciencias forenses, identificación odontológica, odontología forense

\section{ÁREAS TEMÁTICAS}

ciencias forenses; odontología legal y forense

\section{ABSTRACT}

Background: Dental materials, including dental amalgam, undergo macro-structural changes when exposed to high temperatures. Understanding those changes can be useful for dental identification and documentation when conducting medical-legal autopsies of burned, charred, or incinerated bodies to determine type of biomaterial and highest temperature reached. Purpose: Describe the formation of silver nodules on the surface of dental amalgams exposed to high temperatures, in order to identify patterns and reliable markers for forensic dentistry use. Methods: This was a quasi-experimental in vitro study that described the formation of silver nodules in a sample of 45 dental amalgam discs (brands: Contour $₫$ Kerr $₫$, Admix $®$ SDI $₫$, and Nu Alloy ${ }^{\circledR}$ New Stetic () and 45 occlusal fillings in premolars that were exposed to high temperatures $\left(200{ }^{\circ} \mathrm{C}, 400{ }^{\circ} \mathrm{C}, 600{ }^{\circ} \mathrm{C}\right.$, and $\left.800{ }^{\circ} \mathrm{C}\right)$. Results: Silver nodules were evident on the amalgam surfaces between $200{ }^{\circ} \mathrm{C}$ and $400{ }^{\circ} \mathrm{C}$ and began to disappear at $600{ }^{\circ} \mathrm{C}$ to form spheres and strips upon reaching $800{ }^{\circ} \mathrm{C}$. Conclusions: The formation and disappearance of silver nodules is a recurring feature of new-generation amalgam alloys exposed to extreme temperatures. Therefore, describing them can be used as a marker to identify materials and determine highest temperature reached by human remains.

\section{KEYWORDS}

dental amalgam; dental identification; forensic dentistry; forensic sciences; high temperatures; silver nodules

\section{THEMATIC FIELDS}

forensic sciences; legal and forensic dentistry 


\section{INTRODUCCIÓN}

En la investigación científico-criminalística de la muerte, tanto en los procesos de identificación como en la documentación de la necropsia médico-legal, el trabajo de investigadores, fiscales y peritos forenses (incluido el odontólogo) debe integrar la recolección, el análisis, la clasificación y la interpretación de la información obtenida a partir del examen minucioso de los tejidos blandos y duros que conforman el sistema estomatognático (1,2). En el caso de cadáveres o restos humanos quemados, carbonizados o incinerados, el reconocimiento visual y la posibilidad de recuperar las huellas digitales mediante tratamiento de pulpejos es difícil, debido a que las altas temperaturas destruyen la epidermis y los tejidos subyacentes. Por tal razón, diferentes componentes del sistema estomatognático se utilizan para obtener características distintivas e indiciarias que permitan llegar a una identificación positiva a través de métodos odontológicos y antropológicos. Con ellos, en determinado caso, es posible hallar la coincidencia fehaciente de las características físicas del fallecido con los registros de esas mismas características antes de la muerte. En tales casos, el odontólogo establece la identidad por medio de los dientes, al comparar los registros post mortem obtenidos del cadáver o restos humanos con la historia clínica odontológica ante mortem (3-7).

La Junta de Odontología Forense de Estados Unidos (8) clasifica la posibilidad de establecer la identidad de un individuo o sus restos humanos como positiva (coincidencia total), posible (compatibilidad), insuficiente (información disponible inadecuada) o exclusiva (incoherencia e incompatibilidad) (9-11). Ello, a partir de la comparación o cotejo de tratamientos odontológicos, como restauraciones protésicas y obturaciones, dada la gran resistencia que tienen los tejidos dentales y algunos materiales de uso odontológico, incluida la amalgama dental $(6,7)$. Este mismo criterio es utilizado por las leyes nacionales colombianas (Ley 906 de 2004 y Ley 38 de 1994) e internacionales (derechos humanos, Convenio de Ginebra, entre otros).

Entre esos materiales, la amalgama dental se caracteriza por ser biocompatible, tener bajo costo y poseer buenas propiedades físicas y mecánicas; no obstante, carece de adhesión al sustrato dentario, es poco estética y no posee cualidades anticariogénicas. Por tal razón, entre sus indicaciones se encuentra la obturación de cavidades clases Iy ll, luego de remover mecánicamente la caries, y cuando la prioridad se ha centrado en la rehabilitación morfofuncional antes que en la estética (12-14). El método más usado durante los últimos 150 años en el mundo para obturar cavidades terapéuticas de dientes que han sufrido caries ha sido la restauración en amalgama dental, debido a su durabilidad y rentabilidad, y a pesar del desarrollo y uso actual de materiales poliméricos estéticos (15-17).

La amalgama dental es una aleación metálica estable que corresponde a compuestos binarios intermetálicos que interactúan en un proceso en cual que se forma una serie de fases cristalográficas que constituyen la matriz. Ellas se describen como: 1) la fase gamma o compuesto intermetálico de plata y estaño, que no se ha disuelto por el mercurio y que ofrece mayor resistencia a la compresión con pocos cambios dimensionales en el fraguado. 2) La fase gamma 1 o compuesto intermetálico de plata y mercurio, en el que el mercurio entra en la estructura cristalina de la plata para formar una solución sólida $(18,19)$. Es muy resistente a la compresión y presenta gran expansión. Y 3) la fase gamma 2 o compuesto de estaño y mercurio, que presenta las peores propiedades mecánicas y sufre un importante proceso de corrosión (constituida como tal en la restauración disminuye las propiedades mecánicas de la aleación) $(20,21)$.

Si bien en la literatura especializada se han descrito los cambios macroscópicos que ocurren cuando la amalgama dental se somete a altas temperaturas, este estudio in vitro tuvo como objetivo describir la formación de los nódulos de plata en la superficie de discos y obturaciones oclusales en premolares sometidos a la acción de altas temperaturas. El propósito fue identificar patrones y contribuir con el diseño de un marcador fehaciente de utilidad en las ciencias forenses para los procesos de identificación odontológica y documentación de la necropsia médico-legal, en caso de cadáveres o restos humanos quemados, carbonizados o incinerados.

\section{MATERIALES Y MÉTODOS}

Este fue un estudio de corte transversal con diseño cuasi experimental. Se analizó mediante estereomicroscopia el comportamiento in vitro de 45 discos y de 45 obturaciones oclusales en premolares que se elaboraron en tres marcas comerciales de amalgama dentaly que se sometieron a altas temperaturas $\left(200^{\circ} \mathrm{C}\right.$, $400^{\circ} \mathrm{C}, 600^{\circ} \mathrm{C}$ y $800^{\circ} \mathrm{C}$ ). Para estandarizar los cambios se emplearon tres sistemas de amalgama dental en los que sus componentes tuvieran la misma proporción, 
dos de ellos de amplio uso mundialmente (Contour ${ }^{\circledR}$ de Kerr $\circledast$ y Admix ${ }^{\circledR}$ de $S D I \circledast$ ) y uno de amplio uso en Colombia (Nu Alloy ${ }^{\circledR}$ de New Stetic $($ ). Las tres marcas comerciales de amalgama dental empleadas en este estudio son materiales de última generación (tamaño de partícula mixto, esto es, diferente tamaño y alto contenido de cobre para eliminar la fase gama 2) que cuentan con proporciones similares de los metales de la aleación (22). La constitución fundamental es limadura (plata, estaño y cobre) y mercurio (tabla 1 ).

TABLA 1

AMALGAMAS DENTALES EMPLEADAS EN ESTE ESTUdiO

\begin{tabular}{|c|c|c|}
\hline Marca & $\begin{array}{c}\text { Contenido y } \\
\text { proporción (\%) } \\
\text { de los componentes }\end{array}$ & $\begin{array}{c}\text { Casa } \\
\text { comercial, } \\
\text { país }\end{array}$ \\
\hline Contour $\circledR$ & $\begin{array}{l}\text { Plata (41) } \\
\text { Estaño (31) } \\
\text { Cobre (28) } \\
\text { Mercurio (41) }\end{array}$ & $\begin{array}{l}\text { Kerr®, Estados } \\
\text { Unidos }\end{array}$ \\
\hline Admix $®$ & $\begin{array}{l}\text { Plata (49) } \\
\text { Estaño (31) } \\
\text { Cobre }(29) \\
\text { Mercurio }(47,4)\end{array}$ & $\mathrm{SDI}$, Australia \\
\hline Nu Alloy® & $\begin{array}{l}\text { Plata (45) } \\
\text { Estaño (31) } \\
\text { Cobre (24) } \\
\text { Mercurio (50) }\end{array}$ & $\begin{array}{l}\text { New Stetic }(\text {, } \\
\text { Colombia }\end{array}$ \\
\hline
\end{tabular}

\section{Elaboración de los discos en amalgama dental}

Se emplearon una matriz de aluminio, láminas de acetato y losetas de vidrio para elaborar 5 discos de $10 \mathrm{~mm}$ de diámetro por $5 \mathrm{~mm}$ de espesor. Para cada disco se utilizaron 4 cápsulas de una dosis de amalgama dental. Cada cápsula se trituró en un amalgamador Variamix® Dentsply ${ }^{\circledR}$ durante $12 \mathrm{~s}$. Los discos se elaboraron mediante la técnica convencional de empacado (disposición de la amalgama en la matriz), condensado (compactación de la amalgama en la matriz) y bruñido (adaptación de la amalgama respecto al borde cavo de la matriz) $(22,23)$. Finalizada la fase de cristalización, los discos se retiraron de la matriz y se pulieron. Cada espécimen se depositó en un recipiente plástico opaco individual y se mantuvo a una humedad relativa y temperatura ambiente. Antes de exponerlo a las altas temperaturas, a cada espécimen se le tomó una fotografía digital en un estereomicroscopio digital Leuchtturm $®$ de 1,3 megapíxeles a 15X de aumento. Los 54 discos se asignaron, por conveniencia, a cada uno de los 6 grupos (uno de control a temperatura ambiente y 5 de intervención) de acuerdo con los rangos de temperatura (tabla 2).
TABLA 2

DISTRIBUCIÓN DE LA MUESTRA

\begin{tabular}{|c|c|c|c|c|c|c|}
\hline \multicolumn{7}{|c|}{ Discos } \\
\hline \multirow{3}{*}{$\begin{array}{c}\text { Marcas } \\
\text { de } \\
\text { amalgama }\end{array}$} & \multicolumn{6}{|c|}{ Temperatura $\left({ }^{\circ} \mathrm{C}\right)$} \\
\hline & Grupo & \multicolumn{5}{|c|}{ Grupos de intervención } \\
\hline & 28 & 200 & 400 & 600 & 800 & 1000 \\
\hline Contour $\circledR$ & 3 & 3 & 3 & 3 & 3 & 3 \\
\hline Admix $®$ & 3 & 3 & 3 & 3 & 3 & 3 \\
\hline Nu Alloy® & 3 & 3 & 3 & 3 & 3 & 3 \\
\hline \multicolumn{7}{|c|}{ Obturaciones } \\
\hline \multirow{3}{*}{$\begin{array}{c}\text { Marcas } \\
\text { de } \\
\text { amalgama }\end{array}$} & \multicolumn{6}{|c|}{ Temperatura $\left({ }^{\circ} \mathrm{C}\right)$} \\
\hline & $\begin{array}{l}\text { Grupo } \\
\text { control }\end{array}$ & \multicolumn{5}{|c|}{ Grupos de intervención } \\
\hline & 28 & 200 & 400 & 600 & 800 & 1000 \\
\hline Contour® & 3 & 3 & 3 & 3 & 3 & 3 \\
\hline Admix $®$ & 3 & 3 & 3 & 3 & 3 & 3 \\
\hline Nu Alloy® & 3 & 3 & 3 & 3 & 3 & 3 \\
\hline
\end{tabular}

\section{Elaboración de las obturaciones oclusales}

La investigación obtuvo el aval del Comité de Ética en Seres Humanos de la Universidad del Valle, Colombia, que se rige por los principios éticos para las investigaciones biomédicas en humanos consignados en la Resolución 008430 de Colombia (24) y la Declaración de Helsinki (25). Asimismo, recibió la autorización de las directivas de la Escuela de Odontología de la Universidad del Valle y se obtuvo la firma del consentimiento informado por parte de los pacientes.

Se recolectaron premolares extraídos por motivos ortodóncicos en la clínica de cirugía oral de la institución. Inmediatamente después de la extracción, se lavaron con agua no estéril para eliminar residuos de sangre y se introdujeron en un recipiente oscuro con la solución fijadora cloramina T al 5 \% (100 g tosilcloramida sódica diluida en $2 \mathrm{~L}$ de agua destilada) durante una semana. Después se almacenaron en solución salina a $37{ }^{\circ} \mathrm{C}$ con una humedad relativa del $100 \%$ y se cambió la solución salina cada dos semanas, hasta iniciar los procedimientos. Posteriormente, un único operador puso en una base de cera cada uno de los dientes y procedió a hacerles una cavidad oclusal, sin afectar la pulpa dental, con una pieza de alta velocidad (NSK $®$ ), refrigeración constante y fresas de diamante de grano mediofino en forma de pera (Diatech $®$ ). Las cavidades tenían una profundidad de $3 \mathrm{~mm}$, diámetro MD de $3 \mathrm{~mm}$ y diámetro VL de $2 \mathrm{~mm}$ de acuerdo con el protocolo establecido $(26,27)$. 
Una vez se confeccionó la cavidad, se realizó una base cavitaria en ionómero de vidrio (Vitrebond $®$ $3 \mathrm{M}$-ESPE $®$ ) y se obturó con uno de los tres sistemas de amalgama de plata a través de la técnica convencional de empacado (disposición de la amalgama en la cavidad), condensado (compactación de la amalgama en la cavidad), bruñido (adaptación de la amalgama respecto al borde cavo de la cavidad) y pulido de la restauración. Antes de la aplicación de las altas temperaturas, a cada uno de los especímenes se les tomó una fotografía por medio de un estereomicroscopio digital Leuchtturm ${ }^{\circledR}$ de 1,3 megapíxeles a 15X de aumento. Los 54 dientes obturados se distribuyeron de forma aleatoria en cada uno de los seis grupos (uno de control a temperatura ambiente y cinco de intervención) de acuerdo con los rangos de temperatura establecidos (tabla 2).

\section{Aplicación de las altas temperaturas}

El procedimiento se realizó con base en el protocolo técnico-científico establecido en el Departamento de Odontoestomatología de la Universidad de Pavía, Italia (28), y los estudios realizados en la Escuela de Odontología de la Universidad del Valle, Colombia (29). El modelo in vitro planteado en el estudio se llevó a cabo en un horno y no con flama directa, teniendo en cuenta que en los diferentes reportes de la literatura la temperatura máxima alcanzada por el cuerpo humano en combustión es de $800{ }^{\circ} \mathrm{Ca} 1000{ }^{\circ} \mathrm{C}$. Este pico se alcanzó a los 25-30 min, para luego mantenerse alrededor de $500{ }^{\circ} \mathrm{C}$ hasta que se consumía todo el oxígeno, se hubiera reducido a carbón (carbonización) todo el material orgánico o se formaran compuestos de calcio, fosfatos, sílice u otros oligoelementos (incineración) (30). Además, este "efecto de mufla" in vitro es lo que comparativamente harían los tejidos periorales, la musculatura facial, el tejido óseo y los tejidos dentales y periodontales in situ (5).

Los especímenes (discos) de los 4 grupos de intervención para cada temperatura $\left(200{ }^{\circ} \mathrm{C}, 400{ }^{\circ} \mathrm{C}\right.$, $600{ }^{\circ} \mathrm{C}$ y $800^{\circ} \mathrm{C}$ ) se colocaron en bandejas de revestimiento refractario individuales (Cera-Fina ${ }^{\circledR}$ Whip Mix®) para facilitar su manipulación. Se sometieron al calor directo dentro de un horno tipo mufla (Thermolyne $\AA$ ) previamente calibrado para cada grupo de temperatura, iniciando en $28^{\circ} \mathrm{C}$ hasta alcanzar cada una de las temperaturas propuestas, con ascensos escalonados de $10{ }^{\circ} \mathrm{C}$ por minuto. Por ejemplo, se introdujeron en el horno los 9 especímenes (3 discos de cada marca) del grupo de $200{ }^{\circ} \mathrm{C}$, cada uno en su respectiva bandeja, con aumentos de la temperatura de $28{ }^{\circ} \mathrm{C}$ a $200{ }^{\circ} \mathrm{C}$. Se dejó enfriar el horno de nuevo a temperatura ambiente y se procedió a sacar las bandejas con los especímenes. Esto mismo se efectuó para los otros grupos de intervención. El mismo protocolo de aplicación se siguió para los especímenes correspondientes a los premolares con obturaciones en amalgama clase I. Al someterse a altas temperaturas, los materiales de uso odontológico, incluidas las amalgamas dentales, pueden sufrir cambios en el color, textura, fisuras y grietas, fracturas, estabilidad dimensional y estallido (31).

\section{Observación de las muestras}

Antes de describir el comportamiento de los discos y las obturaciones oclusales en amalgama en dientes premolares sometidos a altas temperaturas, 2 de los autores se entrenaron y fueron calibrados en la observación de los cambios macroscópicos superficiales de la amalgama. Específicamente, se estandarizaron en identificar la aparición, el desarrollo y la desaparición de los nódulos de plata en cada marca comercial de amalgama en los diferentes grados de temperatura. Esto se hizo con el fin de controlar sesgos y unificar los criterios de observación. De esta forma, se estimó el grado de confiabilidad mediante la prueba kappa con el programa Stata ${ }^{\circledR}$ 6.0, cuyo resultado determinó la estandarización intraobservador $(0,95$ y 0,92$)$ e interobservador $(0,98$ y 0,95$)$ de los 2 observadores, respectivamente.

\section{Análisis estadístico}

A través del programa SPSS ${ }^{2} 2.0$ de IBM, se analizaron las frecuencias para determinar la prevalencia en porcentaje de los cambios macroscópicos en la muestra. Las variables que se tuvieron en cuenta fueron la aparición, la conformación y la desaparición de los nódulos de plata.

\section{RESULTADOS}

Las tres marcas comerciales (Contour ${ }^{\circledR}$ Kerr ${ }^{\circledR}$, Admix $®$ SDI $₫$ y Nu Alloy $®$ New Stetic $®)$ de amalgama dental tuvieron un comportamiento similar en cada rango de temperatura respecto a la formación y comportamiento de los nódulos de plata, tanto en los discos como en las obturaciones clase I en dientes premolares al ser sometidos a la acción de altas temperaturas (tabla 3). 
TABLA 3

CAMBIOS DE LOS DISCOS Y LAS OBTURACIONES OCLUSALES EN AMALGAMA DENTAL SOMETIDOS A ALTAS TEMPERATURAS (OBSERVADOS EN TODOS LOS DISCOS Y OBTURACIONES OCLUSALES)

\begin{tabular}{|c|c|c|c|}
\hline Característica & Contour ${ }^{\circledR}$ & Admix $®$ & Nu Alloy $®$ \\
\hline \multicolumn{4}{|c|}{$200^{\circ} \mathrm{C}$} \\
\hline Textura & $\begin{array}{l}\text { El aspecto superficial es } \\
\text { poroso }\end{array}$ & $\begin{array}{l}\text { El aspecto superficial } \\
\text { es poroso } \\
\text { Aparecen nódulos de plata } \\
\text { superficiales }\end{array}$ & $\begin{array}{l}\text { El aspecto superficial } \\
\text { es rugoso y poroso }\end{array}$ \\
\hline Fisuras y grietas & No se presentan & No se presentan & No se presentan \\
\hline \multicolumn{4}{|c|}{$400{ }^{\circ} \mathrm{C}$} \\
\hline Textura & $\begin{array}{l}\text { El aspecto superficial } \\
\text { es poroso } \\
\text { Aparecen nódulos } \\
\text { superficiales }\end{array}$ & $\begin{array}{l}\text { El aspecto superficial } \\
\text { es compacto } \\
\text { Se observan nódulos } \\
\text { superficiales }\end{array}$ & $\begin{array}{l}\text { El aspecto superficial } \\
\text { es poroso } \\
\text { Aparecen nódulos superficiales }\end{array}$ \\
\hline Fisuras y grietas & $\begin{array}{l}\text { Se observan poros y fisuras } \\
\text { sobre los nódulos }\end{array}$ & $\begin{array}{l}\text { Se observan poros y fisuras } \\
\text { sobre los nódulos }\end{array}$ & $\begin{array}{l}\text { Se observan poros y fisuras } \\
\text { sobre los nódulos }\end{array}$ \\
\hline \multicolumn{4}{|c|}{$600^{\circ} \mathrm{C}$} \\
\hline Textura & $\begin{array}{l}\text { El aspecto superficial es } \\
\text { compacto y desaparecen } \\
\text { nódulos }\end{array}$ & $\begin{array}{l}\text { El aspecto superficial es } \\
\text { compacto y quedan leves } \\
\text { vestigios de los nódulos }\end{array}$ & $\begin{array}{l}\text { El aspecto superficial es } \\
\text { compacto y desaparecen } \\
\text { nódulos }\end{array}$ \\
\hline Fisuras y grietas & $\begin{array}{l}\text { Se observan fisuras y grietas } \\
\text { en donde quedaban los } \\
\text { nódulos }\end{array}$ & $\begin{array}{l}\text { Se observan fisuras y grietas } \\
\text { en donde quedaban los } \\
\text { nódulos }\end{array}$ & $\begin{array}{l}\text { Se observan fisuras y grietas } \\
\text { en donde quedaban los nódulos }\end{array}$ \\
\hline \multicolumn{4}{|c|}{$800{ }^{\circ} \mathrm{C}$ y $1000^{\circ} \mathrm{C}$} \\
\hline Textura & $\begin{array}{l}\text { Se observa una fase de } \\
\text { aspecto pulverizado y una } \\
\text { fase de aspecto compacto en } \\
\text { forma de pequeñas esferas }\end{array}$ & $\begin{array}{l}\text { Se observa una fase de } \\
\text { aspecto pulverizado y una } \\
\text { fase de aspecto compacto } \\
\text { en forma de betas donde se } \\
\text { encontraban los nódulos }\end{array}$ & $\begin{array}{l}\text { Se observa una fase de aspecto } \\
\text { pulverizado y una fase de } \\
\text { aspecto compacto en forma de } \\
\text { pequeñas esferas }\end{array}$ \\
\hline Fisuras y grietas & Se observa fragmentación & Se observa fragmentación & Se observa fragmentación \\
\hline
\end{tabular}

Con respecto a los discos en amalgama dental, en términos generales, a los $200{ }^{\circ} \mathrm{C}$ se perdió el brillo y la superficie se observó más rugosa. A los $400^{\circ} \mathrm{C}$ aparecieron en la superficie rugosa numerosos nódulos redondeados de aspecto poroso y con presencia de fisuras (estos nódulos en la amalgama Admix ${ }^{\circledR}$ SDI ${ }^{\circledR}$ aparecieron desde los $200^{\circ} \mathrm{C}$ ). A los $600^{\circ} \mathrm{C}$, las amalgamas se observaron sin brillo y de color gris oscuro y presentaban profundas líneas de fractura en el lugar donde se habían presentado los nódulos. De igual forma, se evidenció la pérdida de la estabilidad dimensional por convexidad de la superficie de los discos. A los $800{ }^{\circ} \mathrm{C}$ hubo fragmentación de todos los discos, con fragmentos rugosos, opacos y frágiles que tendían a pulverizarse al contacto, y fragmentos lisos, brillantes y compactos agrupados en estructuras redondeadas (figura 1). 
FIGURA 1

DisCOS ELABORADOS EN AMALGAMA DENTAL SOMETIDOS A ALTAS TEMPERATURAS: A) $200{ }^{\circ} \mathrm{C}$. B) $400{ }^{\circ} \mathrm{C}$. C) $600{ }^{\circ} \mathrm{C}$. D) $800{ }^{\circ} \mathrm{C}$
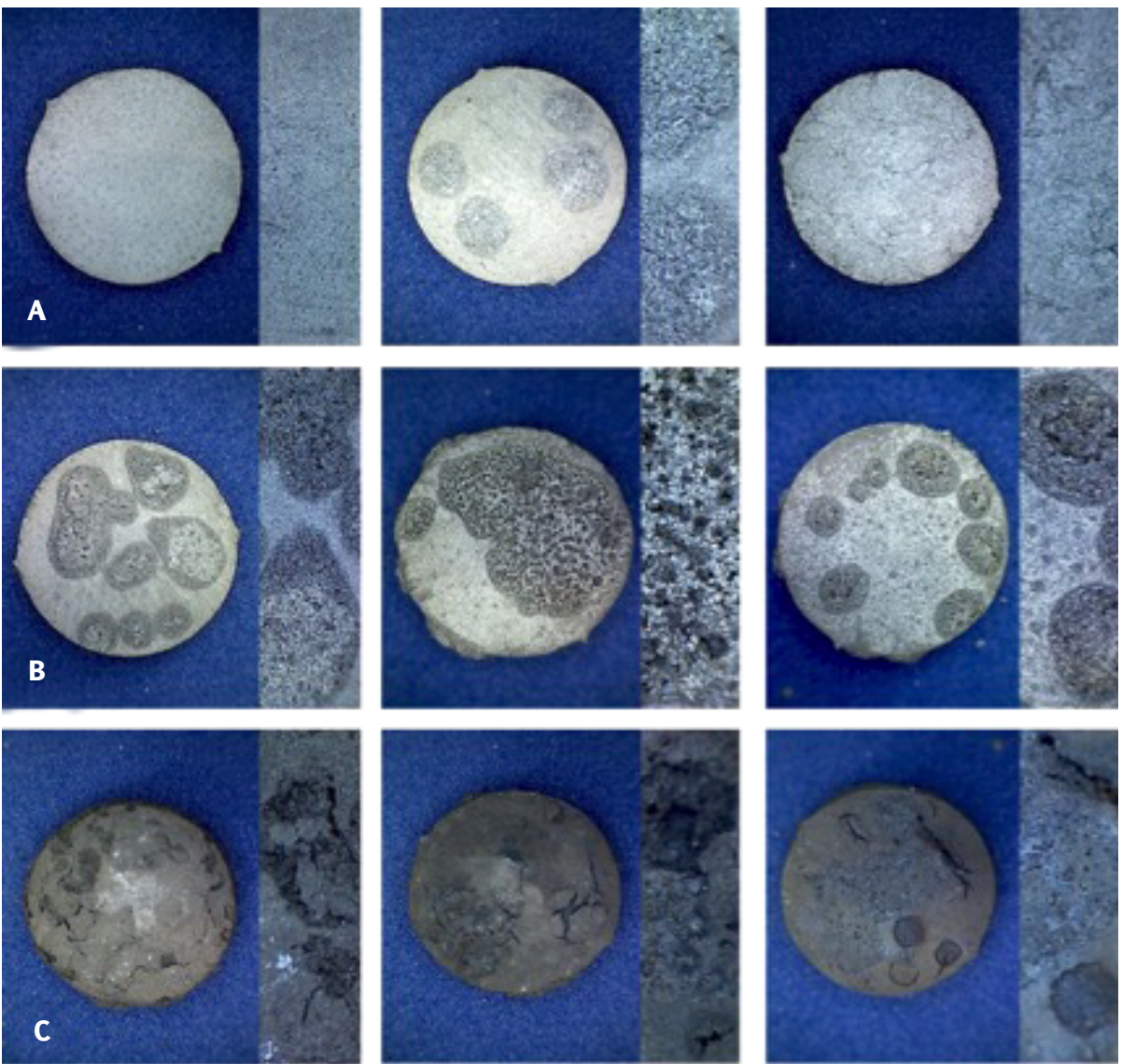

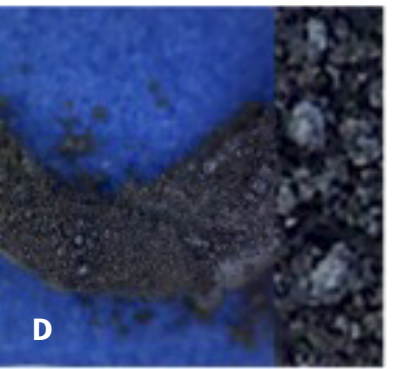

Contour $^{\circledast}$ Kerr $^{\circledast}$

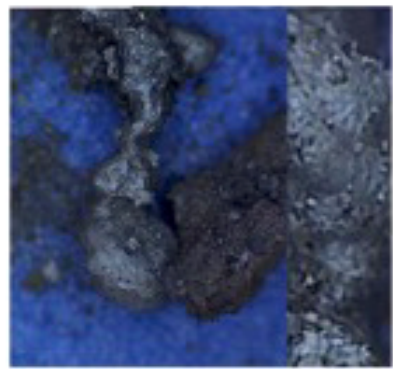

$\left.\operatorname{Admix}{ }^{\circledast S D}\right|^{\circledast}$

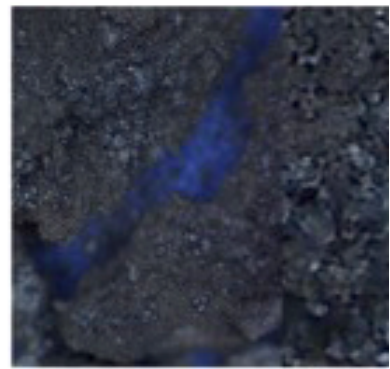

Nu Alloy ${ }^{\circledR}$ Newstethic ${ }^{\circledR}$

En el caso de las obturaciones oclusales en dientes premolares, entre los $200{ }^{\circ} \mathrm{C}$ y los $400{ }^{\circ} \mathrm{C}$ en la superficie de las obturaciones se formaron nódulos de plata. A los $600{ }^{\circ} \mathrm{C}$, pese a la pérdida de las características morfológicas de la obturación, los nódulos se mantuvieron, pero presentaron un aspecto poroso. A los $800^{\circ} \mathrm{C}$, la superficie de la obturación se apreciaba rugosa y fisurada con betas irregulares en el lugar donde quedaban los nódulos (figura 2). 
FIGURA 2

ObTURACIONES OCLUSALES EN AMALGAMA EN PREMOLARES SOMETIDOS A ALTAS TEMPERATURAS:

A) $200{ }^{\circ} \mathrm{C}$. B) $400{ }^{\circ} \mathrm{C}$. C) $600{ }^{\circ} \mathrm{C}$. D) $800{ }^{\circ} \mathrm{C}$
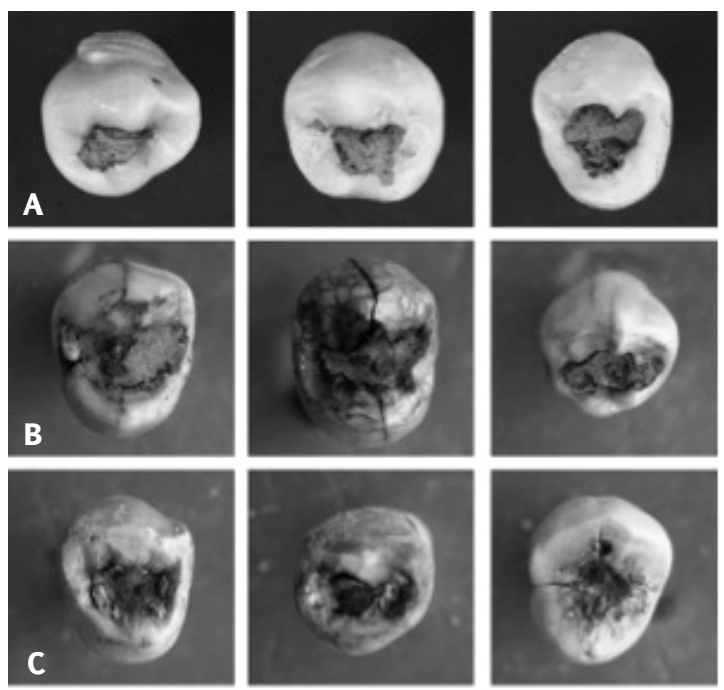

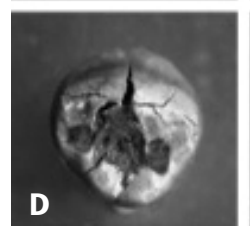

Contour $^{\circledR}$ Kerr $^{\circledR}$

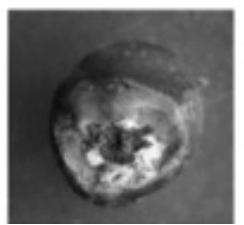

$\operatorname{Admix}^{\circledR} \mathrm{SDl}{ }^{\circledR}$

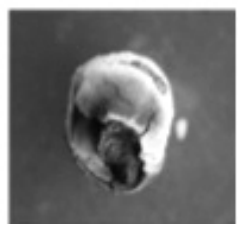

Nu Alloy ${ }^{\circledR}$ Newstethic ${ }^{\circledR}$

\section{DISCUSIÓN}

La formación de nódulos de plata es una característica muy distintiva del comportamiento de la amalgama dental cuando se somete a altas temperaturas. Por tal razón, la discusión de los hallazgos se centra en reconocer su proceso de formación y la manera como este conocimiento podría emplearse en la documentación de los procesos de identificación odontológica y de la necropsia médico-legal. En 2004, Rötzscher y colaboradores (32) citaron a Günther y Schmidt, quienes describieron unas estructuras esféricas, globulares o nodulares en la superficie de las amalgamas sometidas a altas temperaturas. Dichos autores las describieron como "balas de plata" y cuya formación ocurría a partir de una exposición a temperaturas de $800{ }^{\circ} \mathrm{C}$. Este fenómeno se asoció a la evaporación del mercurio. Ello difiere de los resultados de este estudio, ya que la formación de los nódulos plata se detectó desde los $400^{\circ} \mathrm{C}$, con excepción de la amalgama Admix $®$ de SDI® que mostró los nódulos a los $200{ }^{\circ} \mathrm{C}$.

Otros reportes de la literatura especializada forense han descrito el comportamiento de la amalgama dental ante la acción de altas temperaturas y han reportado dichas formaciones. Merlati y colaboradores (28) sometieron a altas temperaturas dientes restaurados con amalgama y encontraron que a partir de los $200{ }^{\circ} \mathrm{C}$ aparecían "burbujas" en la superficie de la amalgama. Igualmente, observaron que a los $600{ }^{\circ} \mathrm{C}$ el mercurio se había evaporado y que únicamente había quedado la limadura. Estos mismos investigadores, en otro estudio, aplicaron altas temperaturas a 75 dientes (25 dientes con obturaciones clase l en amalgama y 25 con obturaciones clase $\mathrm{V}$ en el mismo material). Observaron que en cada grupo de temperatura se presentaban cambios que se repetían en las diferentes muestras, hasta que a los $1000^{\circ} \mathrm{C}$ la amalgama se desintegraba (33). Por su parte, Moreno y colaboradores (31) publicaron un estudio en 2009 en el cual sometieron a altas temperaturas 200 dientes, 50 de los cuales estaban obturados con amalgama dental (GS-80 SDI®). Su objetivo fue determinar, a semejanza del presente estudio, el comportamiento de los dientes obturados al someterlos a altas temperaturas y establecer parámetros aplicables en la identificación odontológica forense en casos restos humanos incinerados. Respecto de la amalgama, los autores describieron que a temperaturas de $200{ }^{\circ} \mathrm{C}$ y $400{ }^{\circ} \mathrm{C}$ este material sufre pérdida de brillo y formación de nódulos en la superficie. A los $600^{\circ} \mathrm{C}$ adquiere un color negro opaco y pierde las características morfológicas conferidas durante el bruñido. A los $800^{\circ} \mathrm{C}$, la textura es rugosa y algunos especímenes se encontraban fragmentados. A diferencia de este estudio, evaluaron la exposición de los dientes a temperaturas de $1000{ }^{\circ} \mathrm{C}$ y $1200{ }^{\circ} \mathrm{C}$, en los cuales observaron fragmentación del material.

Patidar y colaboradores (34) sometieron a la acción de altas temperaturas dientes obturados con diferentes materiales de uso odontológico. Entre ellos, la amalgama dental sometida a temperaturas de $200^{\circ} \mathrm{C}$ presentó una superficie rugosa y fisurada, características que se hicieron más evidentes conforme aumentaba la temperatura. A los $800^{\circ} \mathrm{C}$ se presentan unas estructuras globulares en la superficie de las restauraciones. En un estudio cuasi experimental in vitro más reciente, Aramburo y colaboradores (35) observaron los cambios físicos microestructurales de los tejidos dentales (esmalte, dentina y cemento) y de los materiales de uso odontológico empleados comúnmente en endodoncia al ser sometidos a altas temperaturas. Utilizaron 124 dientes humanos, de los cuales 42 estaban restaurados con amalgama dental (GS-80® SDI $®$ ). Encontraron que a los $200{ }^{\circ} \mathrm{C}$ las amalgamas se tornan opacas y rugosas y se forman nódulos superficiales. Al ser expuestos a 
una temperatura de $400^{\circ} \mathrm{C}$, pierden brillo y se observaron fisuras. A los $600^{\circ} \mathrm{C}$, el color se había tornado negro. A los $800{ }^{\circ} \mathrm{C}$ continuaba el color negro y se presentan nódulos redondeados y fisuras internas. A una temperatura de $1000{ }^{\circ} \mathrm{C}$, las amalgamas se hallaron fragmentadas y pulverizadas. En un estudio publicado en 2011, Moreno y Mejía (36) estandarizaron la técnica para observar dientes sometidos a altas temperaturas a través microscopia electrónica de barrido. En el caso de la amalgama dental, a los $200{ }^{\circ} \mathrm{C}$, los cambios estructurales y de textura superficial estaban relacionados con los puntos de fusión de los metales que conformaban la aleación. A los $400{ }^{\circ} \mathrm{C}$, la amalgama presentaba una superficie oclusal rugosa asociada a la aparición de nódulos que surgían al evaporarse el mercurio a través de burbujas gaseosas, las cuales, al disminuir la temperatura por acción de la presión ambiental, agrupan los otros elementos de la aleación que son arrastrados por el mercurio para constituir dichos nódulos.

En síntesis, en este estudio se explica cómo la amalgama dental sometida a altas temperaturas presenta cambios de textura superficial y estructura que se han relacionado con los puntos de fusión (evaporación en el caso del mercurio) de los compuestos intermetálicos que conforman la aleación. Entre los $200{ }^{\circ} \mathrm{C}$ y los $400{ }^{\circ} \mathrm{C}$, la amalgama presenta una superficie oclusal rugosa asociada a la aparición de nódulos que surgen al evaporarse el compuesto binario mercurio-plata. El menor punto de evaporación del mercurio permite que a estas temperaturas se formen burbujas que arrastran consigo partículas de plata —debido a la apreciable estabilidad de este metal en el mercurio en un rango amplio de concentraciones- hacia la superficie de la amalgama y que luego por la presión y la disminución de la temperatura se agrupan para conformar los nódulos de plata. Es decir, la temperatura afecta la fase gamma 1 de tal forma que el mercurio, mientras pasa de estado líquido a gaseoso $\left(357^{\circ} \mathrm{C}\right)$, arrastra partículas de plata hacia la superficie y, cuando se evapora, la plata conforma un nódulo superficial. Cuando las temperaturas se encuentran entre los $800{ }^{\circ} \mathrm{C}$ y los $1000{ }^{\circ} \mathrm{C}$, la plata alcanza su punto fusión, lo que ocasiona la desaparición de los nódulos y la conformación, una vez baje la temperatura, de pequeñas betas dispersas entre el cobre y el estaño, los cuales se encuentran fragmentados y pulverizados, debido a la oxidación del cobre (desde los $600^{\circ} \mathrm{C}$ ) y a la temprana fusión del estaño (desde los $\left.200{ }^{\circ} \mathrm{C}\right)(37-39)$.
Dado que la distribución de los componentes de los tres sistemas comerciales de la amalgama dental empleados en este estudio es similar, el comportamiento de los discos y de las obturaciones se explica por el comportamiento de los metales que constituyen la aleación. La aparición de los nódulos de plata y su posterior desaparición es una característica constante de las amalgamas dentales de última generación (ricas en cobre) cuando son sometidas a altas temperaturas. Por tal razón, esta descripción se puede emplear durante el cotejo ante mortem-post mortem en el proceso de identificación y en la documentación de la necropsia médico-legal en el caso de cadáveres quemados. Se podrán estimar el tipo de material odontológico y la temperatura aproximada alcanzada por el cuerpo o los restos humanos.

\section{CONCLUSIONES}

Uno de los cambios más característicos de los discos y de las obturaciones en amalgama dental al ser sometida a altas temperaturas es la aparición de nódulos de plata en la superficie, desde su aparición a los $400{ }^{\circ} \mathrm{C}$ (lo cual ocurrió a los $200{ }^{\circ} \mathrm{C}$ para el Admix $(\mathrm{SDI} \circledast$ ), su mantenimiento y la aparición de porosidades y fisuras a los $600{ }^{\circ} \mathrm{C}$, y su posterior desaparición después de los $800{ }^{\circ} \mathrm{C}$ para conformar vetas irregulares.

\section{RECOMENDACIONES}

Se recomienda llevar a cabo estudios complementarios de microscopia electrónica de barrido, de espectrometría de masas y de absorción atómica para identificar la presencia (análisis cualitativo) y la cantidad (análisis cuantitativo) de los componentes de la amalgama dental en los nódulos de plata.

\section{CONFLICTOS DE INTERÉS}

Los autores del artículo hacen constar que no existe, de manera directa o indirecta, ningún tipo de conflictos de interés que pueda poner en peligro la validez de lo comunicado. 


\section{REFERENCIAS}

1. Orjuela CE. Odontología forense. En: Téllez NR, editor. Medicina forense: manual integrado. Bogotá, Colombia: Universidad Nacional de Colombia; 2002.

2. Orjuela CE, Duque MA, Velosa G, Carreño MI, Constantín AE. Guía práctica para el dictamen odontológico forense [documento mimeografiado, versión 3]. Bogotá, Colombia: Instituto Nacional de Medicina Legal y Ciencias Forenses; 2011.

3. Rothwell BR. Principles of dental identification. Dent Clin North Am. 2001; 45(2): 253-70.

4. Norrlander AL. Burned and incinerated remains. En: Bowers CM, Bell GL, editores. Manual of forensic odontology. 3th ed. Colorado Springs, Colorado: American Society of Forensic Odontology; 1997. p. 16-8

5. Delattre VF. Burned beyond recognition: Systematic approach to the dental identification of charred human remains. J Forensic Sci. 2000; 45(3): 589-96.

6. Sweet D. Why a dentist for the identification? Dent Clin North Am. 2001; 45(2): 237-51.

7. Pretty IA, Sweet D. A look at forensic dentistry. Part 1: The role of teeth in the determination of human identity. Br Dental J. 2001; 190(7): 359-66.

8. American Board of Forensic Odontology. Body identification guidelines: American Board of Forensic Odontology, Inc. J Am Dent Assoc. 1994 Sep; 125(9): 1244-6, 1248, 1250

9. Marín NL, Moreno F. Odontología forense: identificación odontológica, reporte de casos. Rev Estomatol, 2003; 11(2): 41-9.

10. Marín L, Moreno F. Odontología forense: identificación odontológica de cadáveres quemados. Reporte de dos casos. Rev Estomatol. 2004; 12(2): 57-70.

11. Moreno F, Moreno S, Marín L. Identificación odontológica forense: revisión de la literatura y reporte de un caso. USTASalud Odontol. 2007; 6(1): 60-6.

12. Chain MC, Baratieri LN. Restauraciones estéticas con resinas compuestas en dientes posteriores. Buenos Aires, Argentina: Panamericana; 2001.

13. Guzmán H. Biomateriales odontológicos de uso clínico. 4a ed. Bogotá, Colombia: Ecoe; 2013.

14. Nocchi $\mathrm{C}$. Odontología restauradora: salud y estética. 2a ed. Buenos Aires, Argentina: Panamericana; 2008.

15. Osborne JW. Amalgam: Dead or alive? Dent Update. 2006; 33(2): 94-8.

16. Yengopal V, Harneker SY, Patel N, Siegfried N. Dental fillings for the treatment of caries in the primary dentition. Cochrane Database Syst Rev. 2009; 15(2): CD004483.

17. Maserejian NN, Hauser R, Tavares M, Trachtenberg FL, Shrader P, McKinlay S. Dental composites and amalgam and physical development in children. J Dent Res. 2012; 91(11): 1019-25.

18. Chudnenkoa K, Pal'yanova G. Thermodynamic properties of $\mathrm{Ag}-\mathrm{Au}-\mathrm{Hg}$ solid solutions. Thermochimica Acta. 2013; 572: 65-70.

19. Liu Y, Wang G, Wang J, Chen Y, Longe Z. Phase equilibria and thermodynamic functions for $\mathrm{Ag}-\mathrm{Hg}$ and $\mathrm{Cu}-\mathrm{Hg}$ binary. Thermochimica Acta. 2013; 547: 83-8.

20. Phillips RW. Skinner's science of dental materials. 9th ed. Philadelphia, Pennsylvania: Saunders; 1992.

21. Craig RG, Powers JM, Wataha JC. Dental materials: properties and manipulation. 7th ed. St Louis, Missouri: Mosby; 2000.

22. Gómez CA, Arismendi JA. Estudio del desempeño preclínico y clínico de una amalgama dental comercial. Rev Fac Odontol Univ Antioq. 2010; 22(1): 63-71.

23. Stratis $\mathrm{S}$, Bryant RW. The influence of modified cavity design and finishing techniques on the clinical performance of amalgam restorations: a 2-year clinical study. J Oral Rehabil. 1998; 25(4): 269-78.

24. República de Colombia, Ministerio de Salud y Protección Social (MSPP). Resolución 008430, por la cual se establecen las normas científicas, técnicas y administrativas para la investigación en salud [internet]. Bogotá, Colombia: MSPP; 4 oct 1993 [citado 7 sep 2015]. Disponible en:http://www.urosario.edu.co/urosario_files/a2a24fb07a-f561-4fcc-b611-affff4374bb7.pdf.

25. Asociación Médica Mundial. Principios éticos para las investigaciones médicas en seres humanos, Declaración de Helsinki [internet]. Helsinki, Finlandia: junio 1964. [citado 7 sep 2015]. Disponible en: http://www. urosario.edu.co/EMCSDocumentos/investigacion/ declaracion_helsinki/.

26. Instituto Colombiano de Normas Técnicas (Icontec). Materiales odontológicos: ensayo de la adhesión a la estructura dental. Norma Técnica Colombiana 4882. Bogotá, Colombia: Icontec; 2000.

27. International Organization of Standardization. Dental materials: Testing of adhesion to tooth structure [internet]. ISO/TS 11405: 2003. [citado 25 Mar 2015] Disponible en: https://www.iso.org/obp/ui/\#iso:std:iso:ts:11405:ed2:v1:en

28. Merlati G, Danesino P, Savio C, Fassina G, Osculati A, Menghini P. Observations of dental prostheses and restorations subjected to high temperatures: experimental studies to aid identification processes. J Forensic Odontostomatol. 2002; 20(2): 17-24.

29. Moreno S, León M, Marín L, Moreno F. Comportamiento in vitro de los tejidos dentales y de algunos materiales de obturación dental sometidos a altas temperaturas con fines forenses. Colomb Med 2008; 39(Supl 1): 28-46. 
30. Symes SA, Dirkmaat DC, Ousley SD, Chapman E, Cabo L. Recovery and interpretation of burned human remains [internet]. Rockville, Maryland: U. S. Department of Justice; 2012 [citado 29 abr 2015]. Disponible en: https://www.ncjrs.gov/pdffiles1/nij/grants/237966.pdf.

31. Moreno S, Merlati G, Marín L, Savio C, Moreno F. Effects of high temperatures on different dental restorative systems: Experimental study to aid identification processes. J Forensic Dent Sci. 2009; 1(1): 17-23.

32. Rötzscher K, Grundmann C, Benthaus S. The effects of high temperatures on human teeth and dentures. Int Poster J Dent Oral Med. 2004; 6(1): Poster 213.

33. Merlati G, Savio C, Danesino P, Fassina G, Menghini P. Further Study of restored and unrestored teeth subjected to high temperatures. J Forensic Odontostomatol. 2004; 22(2): 17-24.

34. Patidar KA, Parwani R, Wanjari S. Effects of high temperature on different restorations in forensic identification: Dental samples and mandible. J Forensic Dent Sci. 2010; 2(1): 37-43.

35. Aramburo J, Zapata A, Zúñiga S, Moreno F. Análisis estereomicroscópico de materiales dentales de uso en endodoncia sometidos a altas temperaturas. Rev Estomatol 2011; 19(2): 8-15.

36. Moreno F, Mejía C. Análisis a través de microscopía electrónica de barrido de dos dientes con tratamiento endodóncico sometidos a altas temperaturas: estudio piloto. Rev Fac Odontol Univ Antioq. 2011; 23(19): 22-36.

37. Odanov Z, Djurdjev M. Investigation of the mechanism of mercury removal from a silver dental amalgam alloy. J Serb Chem Soc 2004; 69(12): 1111-20.

38. Mrowec S, Stokosa A. Oxidation of copper at high temperatures. Oxidation of Metals. 1971; 3: 291-311.

39. Kerl B, Forbeck F. Mercurio. En: Stohmann F, editor. Gran enciclopedia de química industrial. Tomo X. Barcelona, España: Soix; 1956.

\section{CORRESPONDENCIA}

Gustavo Sinisterra-Sinisterra

gusinis52@hotmail.com

Liliana Marín-Jiménez

lilimarin18@hotmail.com

Angélica García

angelicagarcia@javerianacali.edu.co

Sandra Moreno-Correa

smmoreno@javerianacali.edu.co

Freddy Moreno-Gómez

fmorenog@javerianacali.edu.co 